\title{
Focal cortical dysplasia: a review of pathological features, genetics, and surgical outcome
}

\author{
Vincent Y. Wang, M.D., Ph.D., Edward F. Chang, M.D., \\ AND NiCHOlas M. BARbARO, M.D. \\ Department of Neurological Surgery, University of California, San Francisco, California
}

\begin{abstract}
Focal cortical dysplasia (FCD) is found in approximately one-half of patients with medically refractory epilepsy. These lesions may involve only mild disorganization of the cortex, but they may also contain abnormal neuronal elements such as balloon cells. Advances in neuroimaging have allowed better identification of these lesions, and thus more patients have become surgical candidates. Molecular biology techniques have been used to explore the genetics and pathophysiological characteristics of FCD. Data from surgical series have shown that surgery often results in significant reduction or cessation of seizures, especially if the entire lesion is resected.
\end{abstract}

\section{KEY WORDS • focal cortical dysplasia • epilepsy - neuronal migration disorder • surgical outcome}

Focal cortical dysplasia is a significant cause of medically refractory epilepsy. Although aberrant cortical lamination had been described in epilepsy patients in the past, FCD was not extensively studied until Taylor, et al., ${ }^{50}$ in 1971 described a series of 10 epileptic patients with various degrees of cortical dysplasia. The characteristic balloon cells in these lesions were first described in this report. During the last 35 years, developments in imaging, electroencephalography, and electrocorticography have allowed more patients with medically refractory epilepsy to undergo resective surgery. Advances in molecular biology and physiology have led to a better understanding of the genetics and pathophysiological characteristics of FCD. We review the pathological features, pathophysiological characteristics, and genetics of FCD, as well as report on surgical outcomes in patients with FCD.

\section{PATHOLOGICAL AND PATHOPHYSIOLOGICAL FEATURES}

Focal cortical dysplasias are part of an array of disorders described variously as disorders of cortical development, cortical dysplasias, cortical dysgenesis, or neuronal migration disorders. ${ }^{42}$ Classification of these disorders depends on either their pathological characateristics or the proposed origin of the pathological elements (for example, neuronal migration). Histological findings in cortical dysplasia in-

Abbreviations used in this paper: AMPA $=\alpha$-amino-3-hydroxy5-methyl-4-isoxazolepropionic acid; EEG = electroencephalography; FCD $=$ focal cortical dysplasia; $\mathrm{GABA}=\gamma$-aminobutyric acid; GluR = glutamate receptor; $\mathrm{MR}=$ magnetic resonance; $\mathrm{NMDA}=$ $N$-methyl-D-aspartate; NR = NMDA receptor. clude architectural abnormalities such as cortical laminar disorganization and columnar disorganization. More severe forms of FCD are characterized by the presence of abnormal neuronal elements, such as immature neurons, dysmorphic neurons, giant cells, and balloon cells. Immature neurons are round homogeneous cells with large nuclei. ${ }^{42}$ Dysmorphic neurons have distorted cell body, axon, and dendrite morphology caused by the accumulation of neurofilaments within the cytoplasm. ${ }^{44,45}$ Giant cells are increased in size but are normal in shape and do not show an accumulation of neurofilaments. ${ }^{42,47}$ Balloon cells are considered a hallmark of FCD, although they are not present in all patients with FCD. First described by Taylor, et al., ${ }^{50}$ these cells have an eosinophilic cytoplasm and an eccentric nucleus. Immunohistochemical studies have shown that balloon cells have both neuronal and glial characteristics. Many of these cells are immunopositive for vimentin, and others are immunopositive for glial fibrillary acidic protein, neuron-specific enolase, microtubule-associated protein, or neuronal-specific nuclear protein. . $2,44,45,48^{-10}$

\section{CLASSIFICATION OF FCD}

To further classify the pathological features associated with FCD, a panel of epileptologists and neuropathologists devised a classification scheme ${ }^{42}$ in 2004 that has been widely adopted. They distinguished two types of dysplasia based on the presence or absence of dysmorphic neurons or balloon cells. In Type I FCD, there are no dysmorphic neurons or balloon cells. In Type IA, isolated architectural abnormalities, usually laminar or columnar disorganization, are found. Type IB is also characterized by architectural abnormalities, but giant cells or immature neurons are also 
seen. It is important to note that no abnormal cells are present in Type I FCD. On the other hand, abnormal neurons are found in type II FCD. In Type IIA, there are dysmorphic cells but no balloon cells. In Type IIB, both dysmorphic cells and balloon cells are found.

Some correlation exists between the neuropathological findings, clinical presentation, and radiographic findings for these types. Type I FCD can be clinically silent and found in healthy patients. Indeed, autopsy studies have revealed that FCD is found in about $1.7 \%$ of healthy brains. ${ }^{6}$ Some patients with Type I FCD may have cognitive impairment instead of epilepsy. ${ }^{42}$ Magnetic resonance imaging findings associated with Type I FCD may include focal cortical thickening, reduced demarcation of the gray-white matter junction, hyperdensity of gray and subcortical white matter on $\mathrm{T}_{2}$-weighted images, hypodensity of subcortical white matter on $\mathrm{T}_{1}$-weighted images, lobar hypoplasia, and atrophy of the white matter core. ${ }^{1,2,11}$ On the other hand, Type II FCD is more likely to be associated with medically refractory epilepsy. On MR images, these lesions may be seen as focal areas of increased cortical thickness, blurring of the gray-white junction, increased signal intensity on $\mathrm{T}_{2}$ weighted images, or extension of cortical tissue with increased signal intensity from the surface to the ventricle. ${ }^{1-3}$, 8,11,26,32 Although different MR findings are associated with Types I and II FCD, there is significant overlap. Thus, a specific categorization of FCD cannot be made solely on the basis of MR imaging findings and must ultimately be based on histological findings. ${ }^{11}$

\section{Investigation of Mechanisms Leading to Epilesy}

Investigation of the mechanisms by which focal cortical dysplastic lesions cause epilepsy is an active area of research. There is evidence for both an increased excitatory state and decreased inhibition. Molecular characterization of these lesions has suggested several potential mechanisms that can lead to hyperactivity of these lesions. One such mechanism is altered expression of NMDA receptors, which are excitatory glutamate receptors that conduct calcium. They are composed of heterodimers of two families, NR1 and NR2. ${ }^{51}$ Numerous studies have demonstrated that FCD lesions have an elevated expression of NR2A/B subunit proteins and their associated clustering protein PSD95 in the dysplastic neurons and that these substances have a low expression level in normal neuronal tissues. $15,34,37,53-55$ There is some evidence to suggest a correlation between the elevation of NR2A/B subunit expression and the degree of in vivo epileptogenicity. ${ }^{37}$ In one animal model of FCD, an NR2B receptor antagonist has raised the threshold of bicuculline-induced epileptic discharge, suggesting that NR2B subunits may have a functional role in the hyperexcitability of the dysplastic lesions. ${ }^{16}$ Nevertheless, the exact mechanism by which elevation of NR2A/B subunit expression leads to epileptogenicity is still unclear.

Another class of glutamate receptors, AMPA receptors, has also been implicated in the pathogenesis of FCD. AMPA receptors are composed of four subunits (Glu R1-4) and conduct sodium. AMPA receptors interact with NMDA receptors by removing the magnesium blockade on the NMDA receptors. Elevated expression of GluR2/3 subunits has been found in FCD lesions. ${ }^{23}$ Increased GluR4 messenger RNA expression has also shown in FCD neu- rons. ${ }^{15}$ Whether the AMPA receptors contribute to the hyperexcitability through interaction with the NMDA receptors is unclear at this point.

In addition to hyperexcitability, decreased inhibition is also implicated in the pathogenesis of FCD. Early studies have shown that parvalbumin and calbindin D28K immunoreactive neurons, which are inhibitory GABAergic neurons, are absent in dysplastic lesions. ${ }^{19}$ The reduction of GABAergic neurons has been confirmed by Spreafico, et al. ${ }^{46}$ who have shown that the level of glutamic acid decarboxylase, the rate-limiting enzyme for GABA synthesis, is greatly reduced in dysplastic regions resected from human patients. These authors have also reported differences in organization of GABAergic neurons in Types I and II cortical dysplasia. In lesions of Type I cortical dysplasia, which have no giant neurons or balloon cells, a reduction in parvalbumin and glutamic acid decarboxylase immunoreactivity has been found. In contrast, lesions of Type II cortical dysplasia have shown an overall reduction of parvalbumin, but the giant cells are surrounded by terminals that are positive for parvalbumin and glutamic acid decarboxylase. ${ }^{46}$ This reduction of GABAergic neurons has also been seen in experimental models of cortical dysplasia. ${ }^{43}$ In contrast, Calcagnotto, et al., ${ }^{7}$ have not found an overall reduction of glutamic acid decarboxylase expression in Type II dysplastic lesions. Instead, they have described a reorganization of GABAergic neurons, including parvalbumin-positive and calbindin-positive interneurons. In contrast to normal tissue, where these interneurons are found in layer II/III, the interneurons in dysplastic lesions were found to be evenly distributed in all cortical layers. In addition, they have found effects on GABA transport in dysplastic lesions, including reduced expression of GABA transporter. The overall effect of these alterations is reduced inhibitory postsynaptic current frequency in type II cortical dysplasia tissue. Thus, there is evidence for both increased excitation and reduced inhibition in various experimental models, as well as in human cortical dysplasia. The exact role of balloon cells in the production of an epilepsy phenotype is unknown.

\section{GENETICS OF FCD}

Focal cortical dysplasia is thought to be a neuronal migration disorder. It lies at the milder end of the spectrum of neuronal migration disorders that exhibit only focal abnormalities. The other end of spectrum includes diseases such as lissencephaly, in which there is a significant reduction of gyri and thickening of gray matter over a large area of the brain.

Although all of these diseases involve neuronal migration as part of their pathogenesis, the underlying causes of neuronal migration dysfunction in the various disorders may be different. Lissencephaly, periventricular nodular heterotopia, and subcortical band heterotopia (double cortex) are single-gene disorders involving proteins that interact with the cytoskeleton. ${ }^{14}$ Lissencephaly, the most severe neuronal migration disorder, is characterized by a smooth brain surface, marked reduction of gyri, and increased cortical thickness. ${ }^{27}$ Mutations in five different genes (LISI, $D C X, 14-3-3 \epsilon, R E L N$, and $A R X)$ have been shown to cause lissencephaly. ${ }^{27}$ Although mutations in each of these genes have been associated with lissencephaly, a different phenotype exists depending on the gene in which the mutation 
occurs. For example, mutations in DCX and RELN are more likely to lead to anterior cortex involvement, and mutations in $L I S 1,14-3-3 \epsilon$, and $A R X$ are more likely to lead to posterior cortex involvement. ${ }^{27}$ Mutations in $D C X$ lead to a characteristic subcortical band of heterotopic neuronal elements, and hence the name doublecortin has been given to the protein encoded by $D C X .{ }^{27}$ Each of the proteins encoded by these genes has some interaction with the cytoskeleton. The LIS1 protein, for example, interacts with tubulin and suppresses microtubule dynamics. ${ }^{27}$ The DCX protein also has a motif that binds to tubulin and may interact with the LIS1 protein. The protein encoded by RELN, on the other hand, is an extracellular protein that interacts with integrin and lipoprotein receptors, which in turn act on downstream protein kinases that regulate cytoskeletal formation. ${ }^{27}$ Mutations in filaminl have been identified in another class of disease, periventricular nodular heterotopia, which is characterized by differentiated neurons inside misplaced nodules. ${ }^{14}$ Filamin1 protein has been thought to be involved in the formation of filopodia, the cell processes that lead and guidea cell's migration, and disruption of filopodia formation may lead to dysfunction in neuronal migration. ${ }^{14}$ A common theme in all of these disorders is involvement of proteins that interact with or regulate cytoskeleton formation.

Lissencephaly and periventricular nodular heterotopia are the best studied neuronal migration disorders. The mechanisms of other diseases are less well understood. Recent evidence has suggested that mutations in other classes of genes may also cause neuronal migration disorders. For example, mutations in Emx2, which encodes a transcription factor involved in forebrain formation, have been found in patients with schizencephaly, although not in all cases. ${ }^{20}$ Schizencephaly is characterized by clefts extending from the pial surface to the lateral ventricle that are lined with abnormally laminated polymicrogyric cortex. It is unclear how Emx2 mutations lead to neuronal migration problems in these patients.

Both congenital and genetic causes have been proposed for FCD. Some evidence supports a genetic basis for FCD or at least a genetic contribution to its pathogenesis. In two studies, patients with FCD were noted to have a family history of epilepsy. ${ }^{35,36}$ In addition, a positive family history of epilepsy was found to predict an earlier age at onset of seizures in patients with FCD.$^{35}$ Studies that include larger numbers of patients are needed to determine whether FCD has a genetic basis or genetic modifiers.

Overlapping clinical and pathological findings have been noted in brains of patients with tuberous sclerosis and in those of patients with other types of cortical dysplasia. Authors of several interesting papers have suggested that TSC1 is involved in the pathogenesis of FCD.,17 Mutations in TSC1 are known to cause tuberous sclerosis, which, like FCD, is characterized by balloon cells, but also has a number of other clinical features. ${ }^{20}$ Amino acid polymorphisms involving exons 5 and 17 in TSC1 and silent base substitutions in exons 14 and 22 were found in a significantly higher proportion of patients with focal dysplasia, compared with controls. ${ }^{5}$ In addition, loss of $T S C l$ heterozygosity has been noted in focal cortical dysplastic lesions themselves. ${ }^{5}$ This loss of heterozygosity is specific to TSCl, as no genomewide genetic instability has been observed. ${ }^{17}$ It is still unclear how mutation or dysfunction of TSC1 leads to neu- ronal migration disorder. In a recent study, however, the protein encoded by TSC1 was found to be part of the mTOR signaling pathway, a protein kinase pathway involved in cell growth, apoptosis, and cell cycle regulation. ${ }^{31}$ Loss of function of TSC1 affects soma size and dendritic spine formation ${ }^{49}$ If the formation of some of these neuronal processes is involved in neuronal migration, this relationship would explain the role of TSC1 in regulation of neuronal migration; however, the exact mechanism is still unclear.

Other proteins that have been shown to be altered in FCD neurons are the Notch and Wnt pathway proteins. These two signaling pathways are known to be involved in neuronal migration. ${ }^{12}$ It is unlikely that patients with FCD have a mutation in the genes that encode these proteins, as mutations in the genes for the key proteins in these pathways usually have widespread phenotypes; however, mutations in genes for the regulators of these genetic pathways may be one cause of FCD. Indeed, it is thought that one mechanism by which Emx2 mutations cause schizencephaly is through alteration of the Wnt1 expression level. ${ }^{33}$

Indirect evidence from experimental models has suggested that interruption of genetic material can lead to FCD. For example, application of radiation or methylazoxymethanol, which both act to disrupt DNA structure, has been used to create models of FCD.?

There are several reasons why no single genetic mutation has been shown to cause cortical dysplasia. First, FCD represents a rather diverse group of neuron structural abnormalities. As described earlier, FCD can be classified into several types (Types IA, IB, IIA, and IIB) on the basis of histopathological criteria. A second reason is that most surgical series have involved only 20 to 60 patients (see later), and these numbers are too low for detection of most rare events. Third and most important, FCD is unlikely to be associated with a single gene mutation, as is lissencephaly or periventricular nodular heterotopia. Rather, it is likely to be associated with subtle polymorphism mutations in the regulatory elements, or to involve multiple genes, as observed in the case of TSC1. Candidate genes that could cause FCD are those involved in neuronal migration pathways, such as the Notch or Wnt signaling pathways. ${ }^{13}$ Mutations in these genes that lead to a loss of function, however, are usually lethal in the embryo. Thus, it is more likely that hypomorphic mutations, or mutations in the regulatory elements, are associated with FCD. Detecting such mutations is much more difficult and requires studies involving large numbers of patients. New mutation analysis methods and new genome investigations such as the International HapMap Project should shed light on the pathogenesis of FCD.

\section{SURGERY FOR FCD}

Since the report by Taylor, et al., in $1971,{ }^{50}$ FCD has become increasingly recognized as a cause of medically refractory epilepsy. With advances in neuroimaging and electrophysiological monitoring, lesions in FCD can be more accurately identified and are thus more amenable to resection.

Epilepsy remains the most common presentation of FCD. Approximately one half of patients with epilepsy have some form of FCD; ${ }^{6}$ however, some patients with Type I FCD may not show symptoms or may show only mild cognitive impairment. 
Medically refractory epilepsy is the main reason for surgical intervention in patients with FCD. Preoperative neuroimaging usually includes high-resolution MR imaging, which can reveal approximately 60 to $90 \%$ of cortical abnormalities in these patients. ${ }^{4,6,10}$ Characteristic findings include an abnormal gyral pattern, increased cortical thickness, poor gray-white matter differentiation, and increased subcortical signal intensity on $\mathrm{T}_{2}$-weighted and fluid-attenuated inversion-recovery MR images. ${ }^{4,6,11}$ Many patients, especially those with normal MR imaging findings, undergo additional diagnostic procedures. Scalp EEG is frequently used and was one of the more important modalities during early surgical series. Approximately one half to two thirds of patients with abnormal EEG findings have a regional ictal abnormality., ${ }^{4,6}$ In some cases, intracranial subdural recordings, most commonly with grid arrays, are used. Chronic subdural recording allows identification of eloquent cortex, in addition to definition of the epileptogenic region. ${ }^{6}$

The first published description of a surgical series of patients with FCD was by Taylor, et al., 50 in 1971. This seminal study included a total of 10 patients, eight of whom had FCD that was confirmed by histological findings. Six of these patients became seizure free after surgery.

The first large series was described by Palmini, et al., ${ }^{40}$ in 1991. This series included 26 patients with focal neuronal migration disorders, 12 of whom had definitive FCD. Five of the 12 patients had lesions confined to one lobe or one area of the brain, and seven had multilobar involvement. Seven of the 12 patients had complete cessation or excellent control of seizures after surgery. ${ }^{39}$ Seizure control was highly correlated with successful resection of the entire lesion or at least the majority of the abnormal tissue. Complete resection did not necessarily predict cessation of seizures, however, as poor control of seizures remained in some patients despite removal of all of the tissue associated with epileptiform discharges in EEG or electrocorticography findings. In 1995, Palmini, et al.. ${ }^{41}$ further characterized these patients, as well as additional patients from two other institutions. In this series of 34 patients, outcome data were available for 27 patients. An important aspect of this study was the careful documentation of the EEG findings in patients with cortical dysplasia. Approximately two thirds of the patients demonstrated characteristic epileptic discharges, including repetitive electrographic seizure activity, repetitive bursting discharges, and continuous or quasicontinuous rhythmic spiking. In this expanded series with longer follow up, $75 \%$ of the patients who underwent complete resection of regions associated with abnormal electrical activities had a good outcome, and the patients in whom some of these areas remained had relatively poor seizure control. Thus, the concept of combined resection of areas showing radiological and electrophysiological abnormalities was emphasized.

Several reports on surgical series were published in the 1990s. Hirabayshi, et al. ${ }^{24}$ compared seizure control after surgery in patients with FCD and patients with mesial temporal sclerosis. Only six of 17 patients with FCD became seizure free or almost seizure free after the surgery, a significantly worse rate of seizure control, compared with the rate for patients who underwent surgery for mesial temporal sclerosis. Outcome was correlated with the extent of the pathological lesions. Involvement of tissue outside of the temporal or frontal lobes was an independent predictor of poor outcome, whereas frontal lesions and temporal locations were factors favoring a good surgical outcome. Otsubo, et al. ${ }^{38}$ reported on nine children with FCD who underwent resection between 1987 and 1991. Three patients became seizure free after the surgery. There was no conclusion about a relationship between the extent of resection and outcome.Wyllie, et al. ${ }^{52}$ reported results for 30 patients after surgery for migration disorders. Sixteen patients with extratemporal epilepsy were available for follow up, 13 of whom either became seizure free or had greater than $90 \%$ reduction of seizure frequency. In this study, 13 patients had Type I FCD involving the temporal lobe. None of them showed abnormalities on MR images. It is important to note that 10 of these patients became seizure free after surgery and the other three reported significant reductions in seizures. Kuzniecky, et al. ${ }^{30}$ described 11 patients who underwent resection for frontocentral malformations. In 10 of the 11 patients, MR images showed abnormalities. In a group of four patients with frontal involvement, one patient became seizure free and the other three had significant reductions in seizures. Three of the five patients with central lesions demonstrated improvement in seizures after surgery, and the remaining two did not have significant improvement. The authors emphasized that regions of dysplasia could contain normally functioning tissue, which would preclude aggressive resection without production of significant neurological deficits.

In the past few years, descriptions of several additional series of patients who underwent surgery for FCD have been published. It is important to note that many of these studies have had longer follow-up times and have included the use of more advanced imaging techniques, compared with earlier studies. Kloss, et al. ${ }^{28}$ have reported on a series of 68 pediatric patients with FCD. Long-term complete seizure control was achieved in approximately $50 \%$ of the patients, and another $20 \%$ of the patients had excellent seizure control. No significant difference in seizure control after surgery was found between the patients who had Type I FCD and those who had Type II FCD. Again, complete resection of the lesion was the most important factor for complete seizure control. Hong, et al. ${ }^{25}$ have described a series of patients with cortical dysplasia who underwent surgery; nine of these patients had FCD. Complete cessation of seizures was observed in seven of the nine patients after surgery.

Tassi, et al., ${ }^{47}$ have reported on a series of 52 patients with focal dysplasia who underwent surgery. In addition to MR imaging and scalp EEG, stereo-EEG was used in some patients to localize the lesions. Overall, $70 \%$ of the patients who underwent resection experienced excellent outcomes, with cessation of seizures or more than a $90 \%$ reduction in seizure frequency. One interesting observation in this series was that patients with Type II FCD fared better than patients with Type I FCD. Seventy-five percent of the patients with Type II FCD had complete cessation of seizures, compared with $62 \%$ of patients with Type IA FCD and 50\% of patients with Type IB FCD.

Kral, et al., ${ }^{29}$ have described 53 patients in whom a diagnosis of FCD was made, $72 \%$ of whom had complete cessation of seizures after surgery and an additional $4 \%$ of whom had no more than two seizures per year after surgery. Identification of structural abnormalities on MR images 
was key to predicting seizure outcome. Multilobar involvement was identified as a predictor of poor outcome in this study. It is important to note that there was no difference in outcome between the patients with temporal as compared to extratemporal involvement in this series. This finding is probably partly due to the fact that most of the extratemporal involvement was in the frontal lobe.

Bautista, et al., have reviewed their experience with surgical treatment of FCD in adults. In this large series of 55 patients, 36 patients had temporal lesions, 19 had extratemporal lesions, and four had multilobar involvement. Most of the patients (87\%) showed focal lesions on MR images, and approximately $60 \%$ showed additional pathological features such as central nervous system infection, hippocampal sclerosis, or low-grade tumor. Overall, $65 \%$ of these patients became seizure free, and $19 \%$ had significant improvement.

In contrast, in a series of 39 pediatric patients that has been described by Hader, et al. ${ }^{21} 72 \%$ of the patients had good seizure control, although there was no significant correlation between seizure control and imaging characteristics, histopathological findings, or location of the lesions. It is important to note that 16 patients had incomplete resection of the lesion and underwent multiple subpial resections. One half of these demonstrated good seizure control after the procedure. Thus, multiple subpial resections may offer additional benefit, especially for patients in whom the abnormal tissue cannot be resected due to functional considerations.

Cohen-Gadol, et al. ${ }^{10}$ have reported their experience with 22 patients with FCD. As in other series, complete resection with histopathological confirmation of a clean margin predicted good outcome. Ninety-two percent of patients who underwent complete resection became seizure free after the procedure, compared with only $43 \%$ of the patients who had margins still positive for FCD. Of the 22 patients, 20 patients harbored abnormalities identified on MR images. As in other series, the lesion areas with abnor-

TABLE 1

Summary of outcomes in surgical series of patients with FCD

\begin{tabular}{lcc}
\hline \hline \multicolumn{1}{c}{ Authors \& Year } & $\begin{array}{c}\text { No. of } \\
\text { Patients }\end{array}$ & $\begin{array}{c}\text { \% w/ Favorable } \\
\text { Outcome* }\end{array}$ \\
\hline Taylor, et al., 1971 & 8 & 75 \\
Palmini, et al., 1991 & 12 & 58 \\
Hirabayshi, et al., 1993 & 17 & 35 \\
Otsubo, et al., 1993 & 9 & 33 \\
Wyllie, et al., 1994 & 28 & 89 \\
Kuzniecky, et al., 1995 & 11 & 64 \\
Palmini, et al., 1995† & 27 & 41 \\
Hong, et al., 2000 & 9 & 89 \\
Kloss, et al., 2002 & 68 & 70 \\
Tassi, et al., 2002 & 52 & 70 \\
Bautista, et al., 2003 & 55 & 84 \\
Kral, et al., 2003 & 53 & 72 \\
Cohen-Gadol, et al., 2004 & 22 & 73 \\
Hader, et al., 2004 & 39 & 82 \\
Chapman, et al., 2005 & 10 & 32 \\
Hamiwka, et al., 2005 & 31 & 58 \\
overall & &
\end{tabular}

* Favorable outcome includes cessation of seizures and significant reduction (at least $70 \%$ ) of seizure frequency.

$\dagger$ This series included some patients from the 1991 series of Palmini, et al. mal discharge often extended beyond the abnormal area identified on MR images. Fauser, et al., ${ }^{18}$ have correlated the control of seizures after resection with histopathological findings and have found that patients with Type I FCD fared better than those with Type II FCD. Sixty-three percent of patients with Type IA and 55\% of those with Type IB were seizure free after surgery, compared with $43 \%$ of patients with Type IIA and 50\% of those with Type IIB.

Chapman, et al., ${ }^{9}$ have described outcomes for 24 patients who had intractable seizures and normal MR imaging findings. In $42 \%$ of these patients, FCD was found. Overall, $80 \%$ of the patients had a significant reduction in the number of seizures after resection. This finding suggests that many patients with intractable seizures and normal MR imaging findings may have FCD and that resection should still be attempted in these patients, as good outcomes are frequently observed.

Hamiwka, et al., ${ }^{22}$ have reported results of a 10-year follow up of patients who had cortical malformations and underwent resection. Thirty-one of these patients had FCD. Only $32 \%$ of the patients remained seizure free over 10 years. Once again, the extent of resection was the most important factor in determining seizure control outcome.

\section{CONCLUSIONS}

Surgery for FCD can benefit many patients, especially if complete resection of abnormal regions is possible. The preoperative workup should include high-resolution MR imaging, videotelemetry, and EEG. It is important to recognize that MR imaging findings may be normal in some cases and that regions of histological FCD may extend beyond the abnormal areas identified on MR images. Intraoperative monitoring frequently includes electrocorticography, cortical stimulation for motor mapping, and, sometimes, somatosensory evoked potentials if the lesion is near the central sulcus. In recent years, frameless navigation systems have been useful in resection. The best outcomes result when resection of areas with radiological and electrophysiological abnormalities can be achieved. When the results from published descriptions of surgical series were combined, approximately $60 \%$ of patients with FCD experienced good seizure control after resection of the lesion (Table 1). As normal radiological studies do not preclude a diagnosis of cortical dysplasia, only careful and complete histopathological examination of tissue removed from patients with pharmacoresistant epilepsy will help to determine the percentage of cases where FCD exists. Neuroimaging techniques will continue to improve over time and will likely be more useful for identifying the extent of lesions in the future. Coregistration of data from high-resolution MR imaging, EEG, magnetoencephalography, positron emission tomography, and functional MR imaging will allow maximal resection of the abnormal regions with less morbidity.

\section{References}

1. Barkovich AJ, Kjos BO: Nonlissencephalic cortical dysplasias: correlation of imaging findings with clinical deficits. AJNR Am J Neuroradiol 13:95-103, 1992

2. Barkovich AJ, Kuzniecky RI: Neuroimaging of focal malformations of cortical development. J Clin Neurophysiol 13: 481-494, 1996 
3. Bastos AC, Comeau RM, Andermann F, et al: Diagnosis of subtle focal dysplastic lesions: curvilinear reformatting from threedimensional magnetic resonance imaging. Ann Neurol 46: 88-94, 1999

4. Bautista JF, Foldvary-Schaefer N, Bingaman WE, et al: Focal cortical dysplasia and intractable epilepsy in adults: clinical, EEG, imaging, and surgical features. Epilepsy Res 55: 131-136, 2003

5. Becker AJ, Urbach H, Scheffler B, et al: Focal cortical dysplasia of Taylor's balloon cell type: mutational analysis of the TSC1 gene indicates a pathogenic relationship to tuberous sclerosis. Ann Neurol 52:29-37, 2002

6. Bingaman WE: Surgery for focal cortical dysplasia. Neurology 62:S30-S34, 2004

7. Calcagnotto ME, Paredes MF, Tihan T, et al: Dysfunction of synaptic inhibition in epilepsy associated with focal cortical dysplasia. J Neurosci 25:9649-9657, 2005

8. Chan S, Chin SS, Nordli DR, et al: Prospective magnetic resonance imaging identification of focal cortical dysplasia, including the non-balloon cell subtype. Ann Neurol 44:749-757, 1998

9. Chapman K, Wyllie E, Najm I, et al: Seizure outcome after epilepsy surgery in patients with normal preoperative MRI. J Neurol Neurosurg Psychiatry 76:710-713, 2005

10. Cohen-Gadol AA, Ozduman K, Bronen RA, et al: Long-term outcome after epilepsy surgery for focal cortical dysplasia. J Neurosurg 101:55-65, 2004

11. Colombo N, Citterio A, Galli C, et al: Neuroimaging of focal cortical dysplasia: neuropathological correlations. Epileptic Disord 5 Suppl 2:S67-72, 2003

12. Cotter D, Honavar M, Lovestone S, et al: Disturbance of Notch1 and Wnt signalling proteins in neuroglial balloon cells and abnormal large neurons in focal cortical dysplasia in human cortex. Acta Neuropathol 98:465-472, 1999

13. Cotter DR, Honavar M, Everall I: Focal cortical dysplasia: a neuropathological and developmental perspective. Epilepsy Res 36:155-164, 1999

14. Couillard-Despres S, Winkler J, Uyanik G, et al: Molecular mechanisms of neuronal migration disorders, quo vadis? Curr Mol Med 1:677-688, 2001

15. Crino PB, Duhaime AC, Baltuch G, et al: Differential expression of glutamate and GABA-A receptor subunit mRNA in cortical dysplasia. Neurology 56:906-913, 2001

16. DeFazio RA, Hablitz JJ: Alterations in NMDA receptors in a rat model of cortical dysplasia. J Neurophysiol 83:315-321, 2000

17. Fassunke J, Blumcke I, Lahl R, et al: Analysis of chromosomal instability in focal cortical dysplasia of Taylor's balloon cell type. Acta Neuropathol 108:129-134, 2004

18. Fauser S, Schulze-Bonhage A, Honegger J, et al: Focal cortical dysplasias: surgical outcome in 67 patients in relation to histological subtypes and dual pathology. Brain 127:2406-2418, 2004

19. Ferrer I, Oliver B, Russi A, et al: Parvalbumin and calbindinD28k immunocytochemistry in human neocortical epileptic foci. J Neurol Sci 123:18-25, 1994

20. Foldvary-Schaefer N, Bautista J, Andermann F, et al: Focal malformations of cortical development. Neurology 62:S14-S19, 2004

21. Hader WJ, Mackay M, Otsubo H, et al: Cortical dysplastic lesions in children with intractable epilepsy: role of complete resection. J Neurosurg 100:110-117, 2004

22. Hamiwka L, Jayakar P, Resnick T, et al: Surgery for epilepsy due to cortical malformations: ten-year follow-up. Epilepsia 46: 556-560, 2005

23. Hilbig A, Babb TL, Najm I, et al: Focal cortical dysplasia in children. Dev Neurosci 21:271-280, 1999

24. Hirabayashi S, Binnie CD, Janota I, et al: Surgical treatment of epilepsy due to cortical dysplasia: clinical and EEG findings. J Neurol Neurosurg Psychiatry 56:765-770, 1993
25. Hong SC, Kang KS, Seo DW, et al: Surgical treatment of intractable epilepsy accompanying cortical dysplasia. J Neurosurg 93:766-773, 2000

26. Hudson TW, Zawko S, Deister C, et al: Optimized acellular nerve graft is immunologically tolerated and supports regeneration. Tissue Eng 10:1641-1651, 2004

27. Kato M, Dobyns WB: Lissencephaly and the molecular basis of neuronal migration. Hum Mol Genet 12:R89-R96, 2003

28. Kloss S, Pieper T, Pannek H, et al: Epilepsy surgery in children with focal cortical dysplasia (FCD): results of long-term seizure outcome. Neuropediatrics 33:21-26, 2002

29. Kral T, Clusmann H, Blumcke I, et al: Outcome of epilepsy surgery in focal cortical dysplasia. J Neurol Neurosurg Psychiatry 74:183-188, 2003

30. Kuzniecky R, Morawetz R, Faught E, et al: Frontal and central lobe focal dysplasia: clinical, EEG and imaging features. Dev Med Child Neurol 37:159-166, 1995

31. Kwiatkowski DJ, Manning BD: Tuberous sclerosis: a GAP at the crossroads of multiple signaling pathways. Hum Mol Genet 14 Spec No. 2:R251-258, 2005

32. Lee BC, Schmidt RE, Hatfield GA, et al: MRI of focal cortical dysplasia. Neuroradiology 40:675-683, 1998

33. Ligon KL, Echelard Y, Assimacopoulos S, et al: Loss of Emx2 function leads to ectopic expression of Wnt1 in the developing telencephalon and cortical dysplasia. Development 130: 2275-2287, 2003

34. Mikuni N, Babb TL, Ying Z, et al: NMDA-receptors 1 and 2A/B coassembly increased in human epileptic focal cortical dysplasia. Epilepsia 40:1683-1687, 1999

35. Montenegro MA, Guerreiro MM, Lopes-Cendes I, et al: Association of family history of epilepsy with earlier age at seizure onset in patients with focal cortical dysplasia. Mayo Clin Proc 77:1291-1294, 2002

36. Montenegro MA, Guerreiro MM, Lopes-Cendes I, et al: Interrelationship of genetics and prenatal injury in the genesis of malformations of cortical development. Arch Neurol 59: 1147-1153, 2002

37. Najm IM, Ying Z, Babb T, et al: Epileptogenicity correlated with increased N-methyl-D-aspartate receptor subunit NR2A/B in human focal cortical dysplasia. Epilepsia 41:971-976, 2000

38. Otsubo H, Hwang PA, Jay V, et al: Focal cortical dysplasia in children with localization-related epilepsy: EEG, MRI, and SPECT findings. Pediatr Neurol 9:101-107, 1993

39. Palmini A, Andermann F, Olivier A, et al: Focal neuronal migration disorders and intractable partial epilepsy: results of surgical treatment. Ann Neurol 30:750-757, 1991

40. Palmini A, Andermann F, Olivier A, et al: Focal neuronal migration disorders and intractable partial epilepsy: a study of 30 patients. Ann Neurol 30:741-749, 1991

41. Palmini A, Gambardella A, Andermann F, et al: Intrinsic epileptogenicity of human dysplastic cortex as suggested by corticography and surgical results. Ann Neurol 37:476-487, 1995

42. Palmini A, Najm I, Avanzini G, et al: Terminology and classification of the cortical dysplasias. Neurology 62:S2-S8, 2004

43. Roper SN, Eisenschenk S, King MA: Reduced density of parvalbumin-and calbindin D28-immunoreactive neurons in experimental cortical dysplasia. Epilepsy Res 37:63-71, 1999

44. Spreafico R, Battaglia G, Arcelli P, et al: Cortical dysplasia: an immunocytochemical study of three patients. Neurology 50: 27-36, 1998

45. Spreafico R, Pasquier B, Minotti L, et al: Immunocytochemical investigation on dysplastic human tissue from epileptic patients. Epilepsy Res 32:34-48, 1998

46. Spreafico R, Tassi L, Colombo N, et al: Inhibitory circuits in human dysplastic tissue. Epilepsia 41 Suppl 6:S168-S173, 2000

47. Tassi L, Colombo N, Garbelli R, et al: Focal cortical dysplasia: neuropathological subtypes, EEG, neuroimaging and surgical outcome. Brain 125:1719-1732, 2002

48. Tassi L, Pasquier B, Minotti L, et al: Cortical dysplasia: electro- 


\section{Focal cortical dysplasia}

clinical, imaging, and neuropathologic study of 13 patients. Epilepsia 42:1112-1123, 2001

49. Tavazoie SF, Alvarez VA, Ridenour DA, et al: Regulation of neuronal morphology and function by the tumor suppressors Tsc1 and Tsc2. Nat Neurosci 8:1727-1734, 2005

50. Taylor DC, Falconer MA, Bruton CJ, et al: Focal dysplasia of the cerebral cortex in epilepsy. J Neurol Neurosurg Psychiatry 34:369-387, 1971

51. Waxman EA, Lynch DR: N-methyl-D-aspartate receptor subtypes: multiple roles in excitotoxicity and neurological disease. Neuroscientist 11:37-49, 2005

52. Wyllie E, Baumgartner, C, Prayson, R, et al: The clinical spectrum of focal cortical dysplasia and epilepsy. J Epilepsy 7:303-312, 1994

53. Ying Z, Babb TL, Mikuni N, et al: Selective coexpression of NMDAR2A/B and NMDAR1 subunit proteins in dysplastic neurons of human epileptic cortex. Exp Neurol 159:409-418, 1999
54. Ying Z, Bingaman W, Najm IM: Increased numbers of coassembled PSD-95 to NMDA-receptor subunits NR2B and NR1 in human epileptic cortical dysplasia. Epilepsia 45: 314-321, 2004

55. Ying Z, Najm IM: Mechanisms of epileptogenicity in focal malformations caused by abnormal cortical development. Neurosurg Clin N Am 13:27-33, vii, 2002

Manuscript received December 2, 2005.

Accepted in final form December 21, 2005.

Address reprint requests to: Nicholas M. Barbaro, M.D., Department of Neurological Surgery, University of California, San Francisco, 505 Parnassus Avenue, San Francisco, California 94130. email: barbaron@neurosurg.ucsf.edu. 